\title{
Prosection-Aided Demonstrations for Gross Anatomy Lectures: Facilitating Effective Teaching-Time and Self- Guided Dissection Experience for Students
}

\author{
Niladri Kumar Mahato ${ }^{1}$ \\ 'Department of Biomedical Sciences, Ohio University Heritage College of Osteopathic Medicine (OUHCOM) Ohio University, Athens, OH, USA
}

Disclose and conflicts of interest: none to be declared by all authors

\begin{abstract}
Introduction: Effective gross anatomy teaching-time in the medical curriculum has drastically been reduced over the years. Given the demands of delivering complex contents, preserving dissection-based learning experience for students, experimenting with newer teaching and evaluation methods, the stress for time-management is great. This point of view article proposes an approach wherein classical classroom-based lectures preceding formal dissection sessions could be replaced by topic-specific, laboratory-based, prosection-aided demonstration sessions. This approach may benefit students with better conceptualization and retention of the material by reducing cognitive loading, improving outcomes of dissection activities, and by optimizing the utilization of the time allotted to anatomy-teaching in the curriculum.
\end{abstract}

Keywords: Anatomy; Lecture; Dissection; Prosection-demonstration.

\section{Introduction}

The stress for time-management in anatomy teaching is great given the need to accommodate cadaverbased teaching time and to experiment with new teaching and evaluation methods. However, students who dissect and examine anatomical relations in situ, demonstrate better visuospatial understanding of the human body. ${ }^{1-6}$ This point-of-view article proposes an approach wherein the classical lecture system preceding formal dissection sessions may be replaced by laboratory-based prosection-demonstration sessions. This approach may help our students (i) to minimize cognitive loading, (ii) to facilitate better engagement with cadaver dissection, (iii) to ensure optimum use of donated cadavers, and (iv) to enable better utilization of time allotted for anatomy teaching.

Gross-anatomy curriculum, over the last two decades, has seen major cuts in teaching time and even a push to do away with cadaveric dissection., ${ }^{1,-11}$ Paradoxically, as larger number of teaching-methods expert get involved in developing and delivering anatomy courses, less and less time gets allocated to dissection-based learning for students. ${ }^{1,7-10}$ As a member of the anatomy academic fraternity, I think discussing potential solutions for these issues is a priority. As of allocation of pedagogical hours, a great deal of teaching-time in the curriculum gets devoted to didactic lectures. Over the years, power-point presentations, life-like diagrams and digital representation of anatomical structures have undoubtedly gotten better and impressive. However, they still lack the three-dimensional features (and the scale) of working with cadavers. Additionally, the material presented in the lecture system often do not blend seamlessly with the real-life structures explored at the dissection sessions, later by the students. Research studies have proven that spatially complex structures and concepts are better grasped from the cadaver, as compared to two-dimensional lecture images. As such, in terms of a cost-benefit analysis, an imbalance exists between time spent on didactic lectures and that assigned to self-exploratory dissection work.

Arguments relating cadaver-based programs: The arguments forwarded to move away from cadaverbased teaching (dissections/prosection) have included reasons such as unavailability of donated bodies, high costs of laboratory maintenance, expensive labor and technician time, the need for supervised dissection by trained faculty to drive clinically relevant contents, and often, the 'inappropriate' introduction of the concept of death to young students. ${ }^{5}$ On the other hand, proponents of cadaveric dissections have always believed that such programs promote better exploration, conceptualization and retention of anatomical information, and sensitization of perceptions and cultural attitudes toward death, and to develop professionalism and ethics. Education research has shown that active student engagement in dissection-based programs inculcates ownership of learning, critical thinking for problem-solving, leadership qualities, and develops respect in considering cultural differences and diversity within 
the team. ${ }^{5}$ Additionally, awareness of body donation programs and ability to avail one, may fulfil an altruistic desire to be useful after death.

Limitations of classroom-based anatomy lectures: Firstly, most of the illustrative contents used in lectures are two-dimensional, lack depth-perception that is essential for appreciating organ relationships while performing dissection or working on actual body structures in the clinical set-up. Secondly, a major limitation of lecture illustrations is the uniformity of scale of the images presented during a lecture i.e., it fails to capture the scale of sizes between illustration, and the real-life structures. One cannot overemphasize the appreciation of relative comparison of organ-size in-situ, that not only helps to appreciate the structurefunction role of the organ inside the body contour but also help in exploring the structure during dissection. This objective is hardly addressed in conventional lectures. Therefore, one can understand the challenge faced by the students in using the contents from these lectures to appreciate the context in the dissection sessions, following these lectures. Thirdly, lectures often discuss clinical implications of complex threedimensional structures that students may find puzzling to explore in the limited time available during dissection. As such, the transition of information from discussions in the lecture room to the real-life structures seen in the dissection room may not be seamless and result in cognitive loading. ${ }^{12-14}$ On the contrary, discussing a topic on prosected material may work as a template for students to understand the real-life context of discussion better, and later use the knowledge in the dissection room.

Merits of prosection-aided gross anatomy teaching: Working on pre-dissected material has been found to encourage better student engagement in anatomy teaching. Although emotionally not as intense as dissections, prosections have been proven to be a better tool to initiate students to dissection. Unlike logistic requirements for dissections, less facility, low laboratory maintenance, and fewer cadavers can make prosection a cost-effective option. Meticulous and skillful dissection, better preservation of normal tissues (and anatomical variations), protection of contextual surface anatomy, can greatly facilitate conceptual and clinical anatomy learning. Given (i) the advantages of the prosection-based instructions, (ii) the need to protect dissection-experience for our students, and (iii) the growing constraints on available gross anatomy teaching-time, this author suggests improvising conventional lecturing in medical anatomy teaching curriculum. Several anatomy educators have proposed prosection-based gross anatomy teaching method as a viable, focused and time-efficient substitute for a dissection-based practical curriculum where anatomical structures and their relations could be adequately observed in cleanly pre-dissected specimens. ${ }^{2,15-17}$ Properly preserved prosections last a long time and thus can be used repeatedly and accessed for integrated teaching with other curricula in the medical school. However, I think that though a prosection-based method saves time, this approach of teaching may be the second-best option to adopt after dissection, considering the greater impact of dissection-based teaching on student learning achieved by self-guided cadaveric exploration. Experientially, as a medical practitioner and teacher, I believe that cadaver-based teaching is still the best option for learning medical anatomy. Therefore, it is imperative that we preserve this valuable experience for our future students.

The concept of prosection-based gross anatomy demonstration: Big-screen display of prosected material during the proposed demonstration, supplemental power-point slides elucidating the contents, hand-outs on learning objectives, clinical notes, and brief instructions on relevant steps in dissection can all be used as resources to discuss a topic in such a session. Resources presented by the faculty can then be shared with the students to review the progress of their own self-directed dissection. Clinical vignette videos can be created with topicspecific contents and discussed in sessions. This may facilitate the students to early understand the real scale of anatomical structures and will provide them an experience of what is expect during dissections in the laboratory.,12 Scheduling prosection-based demonstration sessions in the laboratory precincts will save change-over time on the part of the students and the staff. Additionally, prosection-lectures / presentations may offer better opportunities for realtime, small group interactions with students to review discussed material thereby reducing student cognitive load of information-processing, off the lecture contents. Demonstrations on prosections, as a prelude to student dissection activities, may help in: (i) better management of dissection-room activities by keeping all students on the same level of progress, feedback and review of their work (Figure 1), ${ }^{5,12,18-20}$ (ii) demonstrating clinical importance of relevant anatomical structures; ${ }^{21}$ (iv) helping students understand their dissection manuals better. ${ }^{2,15-17}$ This method can potentially save time for revisions, encourage group discussion to solve clinical-scenario problems, preferably discussing over prosected/dissected the cadavers. ${ }^{22}$ This strategy should reduce cognitive overload, since the focus area for information gathering and synthesis is limited to the cadaver, enhancing conceptualization of the learnt material engaging limited working-memory. ${ }^{13}$ The demands of a recall based on a back-and-forth switching of attention between lecture-contents gathered from the classroom presentations, and the real structures visualized in the cadaver may 
potentiate increases in cognitive loading. ${ }^{12-14}$ Further, engaging laboratory activities integrated with clinical information, scenarios or problem-solving exercises, videos and other electronic resources like cross sectional imaging may make this prosection-based approach worth exploring. $5,12,18-20$

Additional advantages: As additional advantages, engaging senior students or surgery/allied surgical residents to prepare the prosections for the laboratory would allow them to hone their dissection skills and to improve their own performances in future examinations. Although this proposed approach may call for a steady supply of cadavers, require engagement of skillful dissection teams, necessitate qualified personnel for laboratory maintenance, involve expert anatomists or clinical professionals to deliver prosection-based teaching content, this method could provide students great value-added option to learn their anatomy. Additionally, significant time can be saved by conducting this proposed teaching program in the precincts of the dissection laboratory. Repeated and objective identification and display of anatomical structures should increase confidence in the students to face practical exams and to develop proper contextual orientation of these structure..$^{13,23-25}$ Finally, the proposed approach may help to bridge the gap of understanding between theoretical content and structures explored at the dissection table.

\section{Conclusion}

In summary, reduction in effective teaching-time in medical anatomy may be managed by effective usage of pre-dissection prosection-based instruction. This new approach may be used to phase-out regional, gross-anatomy lectures to save precious anatomy teaching time, and at the same time, to promote and preserve the invaluable cadaver-based anatomy learning experience.

\section{PROSECTION-BASED DEMONSTRATION}

POWER POINT AIDS FOR

in-situ

STRUCTURE

IDENTIFICATION
CLINICAL SCENARIO

OR

VIGNETTE VIDEOS

2

\section{LARGE SCREEN REAL-TIME DEMONSTRAION ON DONOR}

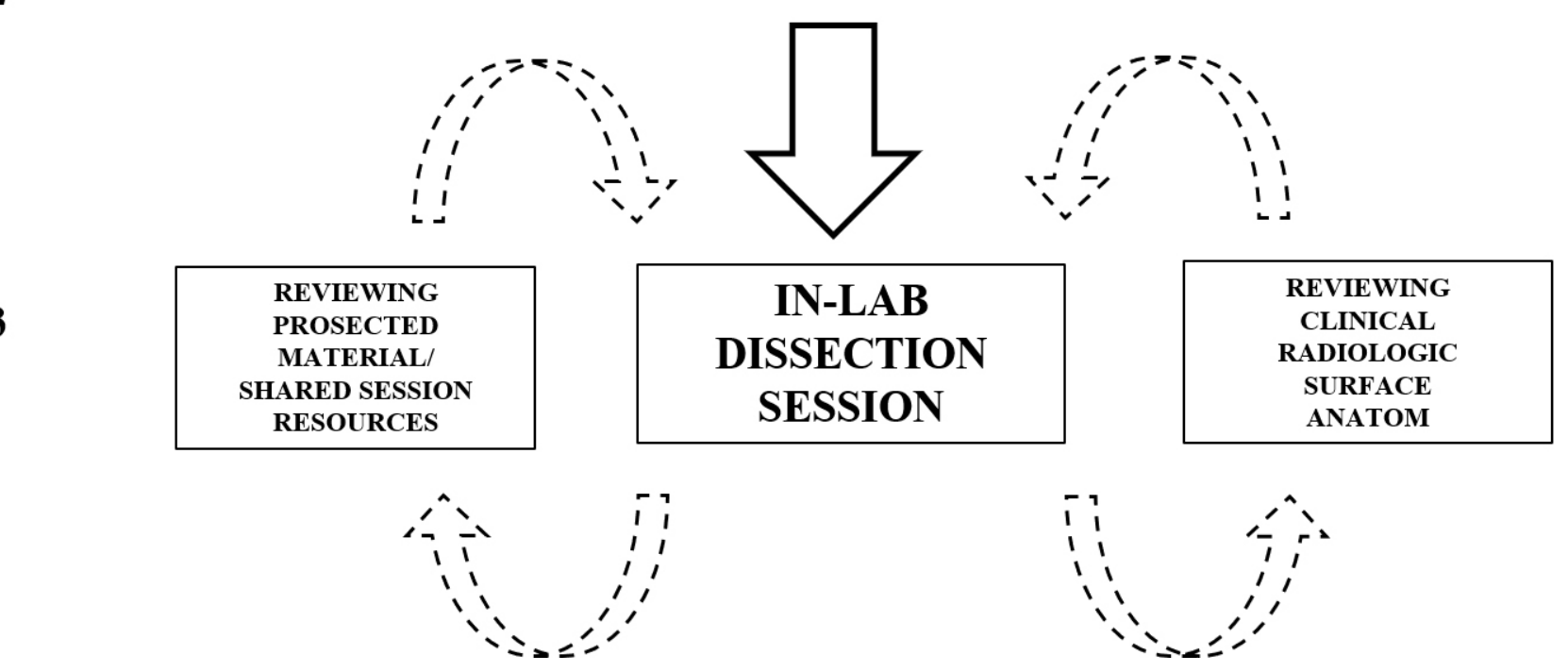

Figure 1. Model of the prosection-based demonstration (upper box) with potential instructional components for the presentation. The dissection lab (lower middle box) follows the demonstration session. Students have the option of reviewing the material discussed from the prosection-session and the available prosected specimen. Both sessions are held within the precincts of the anatomy laboratory. 


\section{References}

1. Parker LM. Anatomical dissection: why are we cutting it out? Dissection in undergraduate teaching. ANZ journal of surgery. 2002;72(12):910-912.

2. Granger NA. Dissection laboratory is vital to medical gross anatomy education. Anatomical record Part B, New anatomist. 2004;281(1):6-8.

3. Pabst R. Gross anatomy: an outdated subject or an essential part of a modern medical curriculum? Results of a questionnaire circulated to final-year medical students. Anat Rec. 1993;237(3):431433.

4. Johnson JH. Importance of dissection in learning anatomy: personal dissection versus peer teaching. Clinical anatomy. 2002;15(1):38-44.

5. Plaisant $\mathrm{O}$, Courtois R, Toussaint PJ, et al. Medical students' attitudes toward the anatomy dissection room in relation to personality. Anatomical sciences education. 2011;4(6):305-310.

6. Dinsmore CE, Daugherty S, Zeitz HJ. Teaching and learning gross anatomy: dissection, prosection, or "both of the above?". Clinical anatomy. 1999;12(2):110-114.

7. Turney BW. Anatomy in a modern medical curriculum. Annals of the Royal College of Surgeons of England. 2007;89(2):104-107.

8. Patel KM, Moxham BJ. Attitudes of professional anatomists to curricular change. Clinical anatomy. 2006;19(2):132-141.

9. Grkovic I, Marinovic Guic M, Kosta V, Poljicanin A, Caric A, Vilovic K. Designing anatomy program in modern medical curriculum: matter of balance. Croatian medical journal. 2009;50(1):49-54. 10. Drake RL, McBride JM, Lachman N, Pawlina W. Medical education in the anatomical sciences: the winds of change continue to blow. Anatomical sciences education. 2009;2(6):253-259.

11. McLachlan JC, Bligh J, Bradley P, Searle J. Teaching anatomy without cadavers. Medical education. 2004;38(4):418-424.

12. Fraser $\mathrm{KL}$, Ayres $\mathrm{P}$, Sweller J. Cognitive Load Theory for the Design of Medical Simulations. Simulation in healthcare : journal of the Society for Simulation in Healthcare. 2015;10(5):295-307. 13. van Merrienboer J, Sweller J. Cognitive load theory in health professional education: design principles and strategies. Medical education. 2010;44(1):85-93.

14. Khalil MK, Paas F, Johnson TE, Payer AF. Interactive and dynamic visualizations in teaching and learning of anatomy: a cognitive load perspective. Anatomical record Part B, New anatomist. 2005;286(1):8-14.

15. Rizzolo LJ, Stewart WB, O'Brien M, et al. Design principles for developing an efficient clinical anatomy course. Medical teacher. 2006;28(2):142-151.

16. Pawlina W, Lachman N. Dissection in learning and teaching gross anatomy: rebuttal to McLachlan. Anatomical record Part B, New anatomist. 2004;281(1):9-11.

17. Yeager VL. Learning gross anatomy: dissection and prosection. Clinical anatomy. 1996;9(1):57-59.

18. Paech D, Giesel FL, Unterhinninghofen R, Schlemmer HP, Kuner T, Doll S. Cadaver-specific CT scans visualized at the dissection table combined with virtual dissection tables improve learning performance in general gross anatomy. European radiology. 2017;27(5):2153-2160.

19. Han ER, Chung EK, Nam KI. Peer-Assisted Learning in a Gross Anatomy Dissection Course. PloS one. 2015;10(11):e0142988.

20. Hofer RE, Nikolaus OB, Pawlina W. Using checklists in a gross anatomy laboratory improves learning outcomes and dissection quality. Anatomical sciences education. 2011;4(5):249-255.

21. Lempp HK. Perceptions of dissection by students in one medical school: beyond learning about anatomy. A qualitative study. Medical education. 2005;39(3):318-325.

22. Vasan NS, DeFouw DO, Holland BK. Modified use of team-based learning for effective delivery of medical gross anatomy and embryology. Anatomical sciences education. 2008;1(1):3-9.

23. Bockers A, Mayer C, Bockers TM. Does learning in clinical context in anatomical sciences improve examination results, learning motivation, or learning orientation? Anatomical sciences education. 2014;7(1):3-11.

24. DiLullo $C$, Coughlin $P$, D'Angelo $M$, et al. Anatomy in a new curriculum: facilitating the learning of gross anatomy using web access streaming dissection videos. Journal of visual communication in medicine. 2006;29(3):99-108.

25. Granger NA, Calleson D. The impact of alternating dissection on student performance in a medical anatomy course: are dissection videos an effective substitute for actual dissection? Clinical anatomy. 2007;20(3):315-321.
Received: June 4, 2019

Accepted: October 18, 2020
Corresponding author

Niladri Kumar Mahato mahato@ohio.edu 